\title{
marcadores de microsatélites en nueve especies de pinos
}

\section{Transferability of microsatellite markers among nine pine species}

\author{
Isaac Sandoval-Padilla1,2, Aremi Rebeca Contreras-Toledo', Luis Felipe Guzmán', \\ Blanca Amalia Amaro González' y Moisés Cortés-Cruz ${ }^{*}$
}

\begin{abstract}
1 Instituto Nacional de Investigaciones Forestales, Agrícolas y Pecuarias. Centro Nacional de Recursos Genéticos. Tepatitlán de Morelos, Jalisco, México.
\end{abstract}

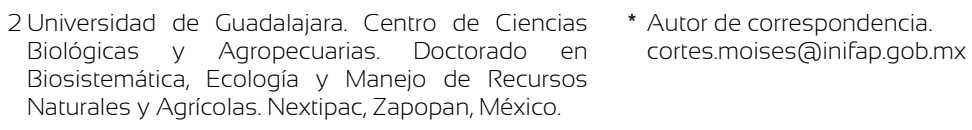

\section{RESUMEN}

En México se encuentran presentes 94 especies de coníferas, de las cuales el género Pinus L. destaca con 49. No obstante, los estudios de diversidad genética de poblaciones mexicanas de pinos son escasos, particularmente aquellos que incluyen marcadores moleculares, basados en secuencias simples repetidas (SSR), considerados especie-específicos, como los microsatélites. El costo inicial para su identificación es elevado cuando no se ha secuenciado el genoma de las especies, así como el diseño de cebadores que permitan la amplificación de las regiones SSR. Sin embargo, una alternativa es la evaluación de los SSRs en especies relacionadas. El objetivo del presente trabajo fue evaluar la transferibilidad de marcadores SSR desarrollados para $P$. taeda en diferentes especies de pinos. Se extrajo ADN genómico de P. ayacahuite, P. cembroides, P. devoniana, P. hartwegii, P. lumboltzii, P. luzmariae, P. patula, P. jeffreyi y P. psendostrobus. basado en el método de CTAB (bromuro de hexadeciltrimetilamonio) a partir de tejido liofilizado Los marcadores fueron seleccionados por grupos de ligamiento (GL), por su motivo de repetición y por su posición dentro de cada GL. Finalmente, los fragmentos amplificados por PCR fueron cuantificados. Treinta y siete marcadores (95\%) amplificaron en al menos una de las nueve especies evaluadas. De ellos, 27 (69\%) presentaron amplificación en más de 50\% de las especies. Estos marcadores presentan cobertura en los 12 GL. Se observó amplificación de más de $75 \%$ en $P$. jeffreyi, $P$. pseudostrobus y $P$. devoniana. La transferibilidad de estos marcadores representa una alternativa para realizar estudios de diversidad genética en especies de pinos.

PALABRAS ClAVE: amplificación interespecífica, diversidad genética, Pinus, SSR.

\section{ABSTRACT}

There are 94 species of conifers distributed in Mexico, from which Pinus L. species are the most abundant with 49 species. Nevertheless, the genetic diversity studies on Mexican populations of pines are limited, particularly those including molecular markers considered species-specific, such as microsatellites (SSR). The initial cost for their identification is high when the species' genome has not been sequenced, as well as the designing of primers that allow the amplification of the SSR regions. However, an alternative is the assessment of SSR in related species. The objective of this study was to evaluate the transferability of SSR markers developed for $P$. taeda in different pine species. Genomic DNA was isolated from P. ayacahuite, P. cembroides, P. devoniana, P. hartwegii, P._lumboltrii, P. luzmariae, P. patula, P. jeffreyi and $P$. pseudostrobus, based on CTAB method (ionic detergent cetyltrimethylammonium bromide) from liophylized tissue. The markers were selected based on linkage group (LG), motif and position within the LG. Finally, fragments were amplified by PCR and products were quantified. Thirty-seven markers (95\%) amplified in at least one of the nine species. From those, $27(69 \%)$ amplified in more than $50 \%$ of the species. These markers were present in the $12 \mathrm{LG}$. Amplification of more than $75 \%$ was observed for P. jeffreyi, $P$. pseudostrobus and P. devoniana. The transferability of these markers represents an alternative for genetic diversity studies across pine species.

KEYWORDS: inter-specific amplification, genetic diversity, Pinus; SSR. 


\section{INTRODUCCIÓN}

Las coníferas son un grupo de árboles y arbustos de gran importancia económica y ecológica, ya que son los componentes dominantes de diversos tipos de vegetación. En México, se encuentran presentes 10 de los 71 géneros del grupo, con 94 especies, de las cuales, 43 son clasificadas como endémicas. Su distribución nacional ocurre desde el nivel del mar hasta los $4000 \mathrm{~m}$ s.n.m. Dentro de las coníferas, el género Pinus destaca con 49 especies (Gernandt y Pérez-de la Rosa, 2014).

Las especies del género Pinus han disminuido en número de poblaciones, por lo que, algunas de ellas se encuentran amenazadas o bajo protección. Esto es resultado de la tala indiscriminada, la pérdida del hábitat debido al cambio de uso de suelo para producción agrícola, el cambio climático que trae como consecuencia la reducción del área de distribución potencial por cambios en la temperatura, sequías y otros eventos extremos incendios forestales, incidencia de plagas y enfermedades y otros fenómenos naturales (Del Castillo, Pérez-de la Rosa, Vargas y Rivera, 2004). Proyecciones en P. hartwegii Lindley, por ejemplo, indican una reducción del hábitat de $86 \%$ para 2050, debido a los efectos del cambio climático (Gutiérrez y Trejo, 2014).

La drástica disminución poblacional puede afectar negativamente los niveles de diversidad genética, implicando una baja capacidad de adaptación al ambiente y hacer frente a factores adversos, acelerando el proceso de extinción (Rocha y Gasca, 2007). Esto es consecuencia del manejo antropogénico en la composición genética y genotípica de las poblaciones vegetales (Freeman y Herron, 2002). Asimismo, la baja diversidad genética y la ausencia de estructura genética en las especies puede ser un reflejo de cambios demográficos a lo largo de las generaciones (Freeman y Herron, 2002; Moreno, 2007). Lo anterior puede estar ocurriendo en las especies del género Pinus debido a la sobreexplotación, la disminución poblacional y la reducción del área de distribución, por lo que los análisis de la diversidad genética son indispensables. A pesar de ello, en poblaciones mexicanas de Pinus estos estudios son escasos, particularmente aquellos que incluyen marcadores moleculares considerados especie-específicos, como los microsatélites o secuencias simples repetidas (SSR). Los SSR son secuencias de ADN formadas de uno a seis pares de bases, encontrándose en regiones codificantes y no codificantes. Estos marcadores son utilizados para estudios de caracterización genotípica en plantas por ser abundantes en el genoma, altamente informativos, multialélicos, codominantes y permiten su transferibilidad entre especies relacionadas (Mason, 2015; Carneiro, Santini, Lima y de Freitas, 2016). Este tipo de marcadores moleculares de ADN permiten inferir la diversidad y estructura genética, tamaños efectivos y mínimos variables de poblaciones para conservación (in situ o ex situ) y selección de genotipos para mejoramiento, eventos de cuellos de botella, cartografía genética, etc. (Rocha y Gasca, 2007; Geburek y Konrad, 2008). Sin embargo, la principal desventaja de estos marcadores es el costo de su generación cuando no se tiene información del genoma de la especie de interés, así como del diseño de cebadores que permitan la amplificación de las regiones SSR (Rentaría, 2007; Rocha y Gasca, 2007; Valdés-Infante Herrero et al., 2010; Carneiro et al., 2016). Una alternativa es la evaluación de SSRs desarrollados para especies relacionadas a la especie de interés (transferibilidad) (Echt, Vendramin, Nelson y Marquardt, 1999; Chagne et al., 2004; Celiński, Pawlaczyk, WojnickaPółtorak, Chudzińska y Prus-Głowacki, 2011; Lesser, Parchman y Buerkle, 2011; Feng, Yang, Wang, Luo y Li, 2014; Villalobos-Arámbula, Pérez de la Rosa, Arias y Rajora, 2014; Rai, Ginwal y Saha, 2018). Así, se pueden identificar marcadores útiles reduciendo el tiempo y consumo de recursos en estudios genéticos para diversas especies de pinos (Villalobos-Arámbula et al., 2014).

\section{OBJETIVOS}

El objetivo del presente trabajo fue evaluar la transferibilidad de marcadores SSR desarrollados para $P$. taeda en nueve especies de pinos. 


\section{MATERIALES Y MÉTODOS}

\section{Material vegetal y extracción de ADN}

Para la extracción de ADN, se emplearon las acículas de nueve especies del género Pinus: P. cembroides Zucc. (San José de Gracia, Aguascalientes), P. devoniana Lindl. (Tapalpa, Jalisco), P. mzrmariae Pérez de la Rosa (Bosque La Primavera, Jalisco), P. patula Schltdl. \& Cham. (Cruz Blanca. Veracruz), P. ayacabuite C. Ehrenb. ex Schltdl. (San Antonio Buenavista, Puebla), P. hartwegii Lindl. (Tapalpa, Jalisco), P. jeffreyi Balf. (Ejido Sierra de Juárez, Baja California), P. lumboltżii B.L. Rob. \& Fernald (Sierra Escondida, Aguascalientes) y $P$. pseudostrobus Brongn (San Cristóbal de las Casas, Chiapas). El proceso de extracción de ADN se realizó basado en el método de CTAB (bromuro de hexadeciltrimetilamonio) a partir de tejido liofilizado. Para las primeras cuatro especies se empleó la misma metodología que Saghai-Maroof, Soliman, Jorgensen y Allard, (1984); para el resto de las especies se siguió la metodología establecida por Huang, Guang, Kong, Guo y Guo, (2013). La concentración y calidad del ADN fue determinada mediante espectrofotometría (NanoDrop 2000, Thermo Scientific) y corroborada mediante electroforesis en geles de agarosa, comparando el patrón de migración de las muestras con el del marcador de referencia, lambda sin cortar (New England Biolabs). Se emplearon tres árboles para cada especie, excepto para las especies $P$. devoniana Lindl. y $P$. hartwegii Lindl, para las cuales solamente se emplearon dos árboles.

\section{Selección de marcadores SSR}

Se realizó la selección de los marcadores de SSR desarrollados para P. taeda (Echt et al., 2011) con base en tres criterios: a) por grupo de ligamiento (GL) (marcadores distribuidos en todos los grupos de ligamiento de las especies del género Pinus), b) motivo de repetición (marcadores con repetición simple de dos o tres nucleótidos) y c) posición genética dentro de cada GL (marcadores ubicados en el brazo corto y largo). Se seleccionaron 39 marcadores, tres por cada uno de los 12 grupos de ligamiento y tres marcadores que no tienen asignación reportada (Tabla 1).

\section{Amplificación por PCR y análisis}

Las reacciones de amplificación mediante PCR se realizaron en un volumen final de $10 \mu \mathrm{L}$ conteniendo $40 \mathrm{ng}$ de $\mathrm{ADN}$, $1 \mu \mathrm{M}$ de cada iniciador y $0.8 \mathrm{X}$ de REDTaq ${ }^{\circledR}$ ReadyMix ${ }^{\mathrm{TM}}$ PCR Reaction Mix (Sigma- Aldrich). Las condiciones de amplificación fueron: una desnaturalización inicial a $95^{\circ}$ por 5 min., seguida de 35 ciclos de desnaturalización a $95^{\circ}$ por 40 $\mathrm{s}$, hibridación a $60^{\circ}$ por $40 \mathrm{~s}$ y elongación a $72^{\circ}$ por 2 min y una de extensión final a $72^{\circ}$ por $10 \mathrm{~min}$. La amplificación se realizó en un termociclador Veriti 96 Well Thermal Cycler (Applied Biosystems). Los productos de la amplificación fueron separados mediante electroforesis en geles de agarosa a $2 \%$ previamente teñidos con GelRed ${ }^{\mathrm{TM}}$ Nucleic Acid Gel Stain (Biotium) en amortiguador TBE 1 X a 120 V constantes durante 2 horas. La visualización de los fragmentos amplificados se realizó exponiendo los geles a luz UV en tanto que la captura de la imagen se realizó mediante el sistema Gel Logic (KODAK Carestream). Finalmente, se realizó el registro y cuantificación de fragmentos para determinar su tamaño y el porcentaje de amplificación.

\section{RESULTADOS Y DISCUSIÓN}

La amplificación de fragmentos de los 39 marcadores SSR fue variable. Treinta y siete (95\%) amplificaron en al menos una de las nueve especies evaluadas. De ellos, 69.27\% (27) presentó amplificación mayor a $50 \%$ a través de las especies, los cuales se encuentran distribuidos en los 12 grupos de ligamiento (GL). Diez de los marcadores (25.64\%) amplificó entre 10\% y 50\% de las especies (Tabla 2). Los marcadores SsrPt_ctg7141, PtRIP_0630 y PtSIFG_1062 amplificaron en las especies $P$. jeffreyi y $P$. psendostrobus mientras que el marcador PtRIP_1072 amplificó exclusivamente en la especie $P$. lumboltziii. Los marcadores PtTX3027 y PtTX3019 no amplificaron en ninguna especie. Los marcadores PtRIP_9315, PtSIFG_0265, PtTX3029, PtTX3045, PtSIFG_0440, PtSIFG_1018, PtSIFG_0249 (17.95\%) amplificaron en todas las especies, localizados en los grupos de ligamiento (GL) 2, 4, 5, 6, 8, 11 y uno sin mapear. 
TABLA 1. Marcadores de microsatélites e iniciadores desarrollados para P. taeda (Echt et al., 2011) utilizados para su amplificación en Pinus spp.

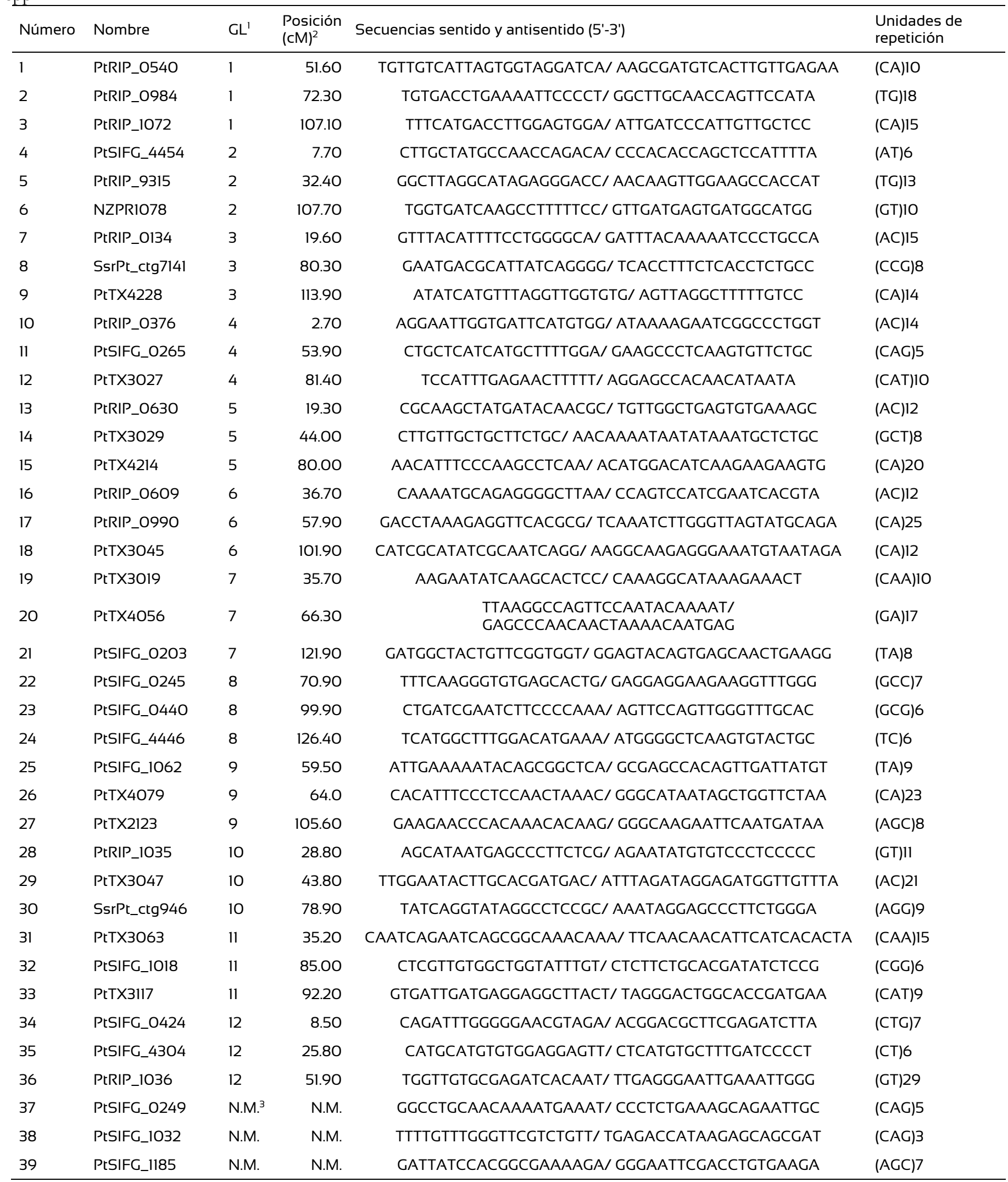


TABLA 2. Amplificación de 39 marcadores de microsatélites en nueve especies de Pinus L. El valor "1" indica amplificación y “0” no amplificación.

\begin{tabular}{|c|c|c|c|c|c|c|c|c|c|c|}
\hline Marcador & 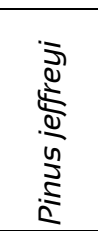 & 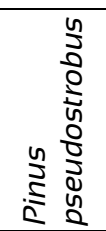 & 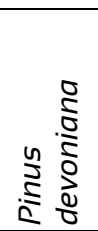 & 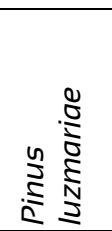 & 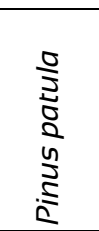 & 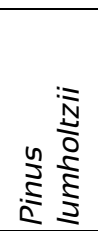 & 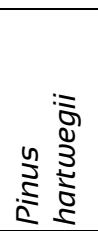 & 量 & 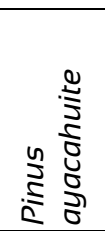 & Amplificación (\%) \\
\hline PtRIP_9315 & 1 & 1 & 1 & 1 & 1 & 1 & 1 & 1 & 1 & 100 \\
\hline PtSIFG_0265 & 1 & 1 & 1 & 1 & 1 & 1 & 1 & 1 & 1 & 100 \\
\hline РtTХ3029 & 1 & 1 & 1 & 1 & 1 & 1 & 1 & 1 & 1 & 100 \\
\hline PtTX3045 & 1 & 1 & 1 & 1 & 1 & 1 & 1 & 1 & 1 & 100 \\
\hline PtSIFG_0440 & 1 & 1 & 1 & 1 & 1 & 1 & 1 & 1 & 1 & 100 \\
\hline PtSIFG_1018 & 1 & 1 & 1 & 1 & 1 & 1 & 1 & 1 & 1 & 100 \\
\hline PtSIFG_0249 & 1 & 1 & 1 & 1 & 1 & 1 & 1 & 1 & 1 & 100 \\
\hline PtTX4056 & 1 & 1 & 1 & 1 & 1 & 1 & 1 & 1 & 0 & 88.89 \\
\hline PtSIFG_0424 & 1 & 1 & 1 & 1 & 1 & 1 & 1 & 1 & 0 & 88.89 \\
\hline PtRIP_0376 & 1 & 1 & 1 & 1 & 1 & 1 & 1 & 0 & 1 & 88.89 \\
\hline PtTX2123 & 1 & 1 & 1 & 1 & 1 & 1 & 1 & 0 & 1 & 88.89 \\
\hline PtSIFG_4304 & 1 & 1 & 1 & 1 & 1 & 1 & 0 & 1 & 1 & 88.89 \\
\hline PtSIFG_1032 & 1 & 1 & 1 & 1 & 1 & 1 & 1 & 1 & 0 & 88.89 \\
\hline PtSIFG_1185 & 1 & 1 & 1 & 1 & 1 & 1 & 1 & 1 & 0 & 88.89 \\
\hline PtRIP_0984 & 1 & 1 & 1 & 1 & 1 & 1 & 1 & 0 & 0 & 77.78 \\
\hline PtSIFG_0245 & 1 & 1 & 1 & 1 & 1 & 1 & 1 & 0 & 0 & 77.78 \\
\hline PtRIP_1036 & 1 & 1 & 1 & 1 & 1 & 1 & 1 & 0 & 0 & 77.78 \\
\hline PtSIFG_4454 & 1 & 1 & 1 & 1 & 1 & 0 & 1 & 0 & 1 & 77.78 \\
\hline SsrPt_ctg946 & 1 & 1 & 1 & 1 & 1 & 0 & 0 & 1 & 1 & 77.78 \\
\hline PtRIP_0540 & 1 & 1 & 1 & 1 & 1 & 1 & 0 & 0 & 0 & 66.67 \\
\hline PtRIP_0134 & 1 & 1 & 1 & 1 & 1 & 0 & 0 & 1 & 0 & 66.67 \\
\hline PtSIFG_4446 & 1 & 1 & 1 & 1 & 1 & 0 & 1 & 0 & 0 & 66.67 \\
\hline PtRIP_1035 & 1 & 1 & 1 & 1 & 0 & 1 & 1 & 0 & 0 & 66.67 \\
\hline РtTX3063 & 1 & 1 & 1 & 1 & 1 & 0 & 1 & 0 & 0 & 66.67 \\
\hline NZPR1078 & 0 & 0 & 1 & 1 & 1 & 1 & 1 & 0 & 0 & 55.56 \\
\hline PtTX4214 & 1 & 1 & 0 & 0 & 1 & 1 & 0 & 1 & 0 & 55.56 \\
\hline РtTX3047 & 1 & 0 & 1 & 1 & 1 & 1 & 0 & 0 & 0 & 55.56 \\
\hline PtRIP_0609 & 0 & 0 & 1 & 1 & 1 & 1 & 0 & 0 & 0 & 44.44 \\
\hline PtRIP_0990 & 0 & 0 & 1 & 1 & 1 & 1 & 0 & 0 & 0 & 44.44 \\
\hline PtSIFG_02O3 & 1 & 1 & 0 & 0 & 0 & 1 & 0 & 1 & 0 & 44.44 \\
\hline PtTX4079 & 0 & 0 & 1 & 1 & 0 & 1 & 1 & 0 & 0 & 44.44 \\
\hline PtTX4228 & 0 & 0 & 1 & 0 & 1 & 1 & 0 & 0 & 0 & 33.33 \\
\hline PrTX3117 & 1 & 1 & 0 & 0 & 0 & 1 & 0 & 0 & 0 & 33.33 \\
\hline SsrPt_ctg7141 & 1 & 1 & 0 & 0 & $\mathrm{O}$ & 0 & 0 & 0 & 0 & 22.22 \\
\hline PtRIP_0630 & 1 & 1 & 0 & 0 & 0 & 0 & 0 & 0 & 0 & 22.22 \\
\hline PtSIFG_1062 & 1 & 1 & 0 & $\mathrm{O}$ & $\mathrm{O}$ & 0 & 0 & 0 & $\mathrm{O}$ & 22.22 \\
\hline PtRIP_1072 & 0 & 0 & 0 & 0 & 0 & 1 & 0 & 0 & 0 & 11.11 \\
\hline РtTX3027 & 0 & 0 & 0 & $\mathrm{O}$ & 0 & 0 & 0 & 0 & $\mathrm{O}$ & 0 \\
\hline PtTX3019 & 0 & 0 & 0 & 0 & 0 & 0 & 0 & 0 & 0 & 0 \\
\hline Transferibilidad (\%) & 79.49 & 76.92 & 76.92 & 74.36 & 74.36 & 74.36 & 56.41 & 41.03 & 30.77 & \\
\hline
\end{tabular}


Se observó un porcentaje de amplificación mayor a 30\% en todas las especies. El mayor porcentaje de transferibilidad se observó en $P$. jeffreyi con 31 marcadores (79.49\%), seguido de $P$. devoniana y $P$. pseudostrobus con 30 marcadores (76.92\%). P. luzmariae, P. patula y P. lumboltziii, por otro lado, mostraron amplificación de 29 marcadores (74.36\%), mientras que $P$. bartwegii, $P$. cembroides y $P$. ayacabuite mostraron amplificación en 22 (56.41\%), 16 (41.03\%) y 12 (30.67\%) marcadores, respectivamente. Siete especies analizadas en el presente estudio tuvieron una transferibilidad de marcadores SSR mayor a 50\%. Estas especies, así como P. taeda, pertenecen a la sección Trifoliae del subgénero Pinus. Por el contrario, las especies con menor transferibilidad ( $P$. ayacabuite y $P$. cembroides) pertenecen al subgénero Strobus secciones Quinquefoliae y Parrya, respectivamente, siendo filogenéticamente más lejanas a P. taeda (Tabla 3). Las regiones complementarias de los iniciadores pudieron haber sufrido algún evento mutacional que impidió en ellos el proceso de hibridación durante el proceso de amplificación por PCR, con la consecuente ausencia de ampliaciones (Chapuis y Estoup, 2007).

Estas tasas de transferibilidad son similares a las observadas en marcadores SSR de P. strobus evaluados en cinco especies de pinos (Villalobos-Arámbula et al., 2014), para las cuales se registró amplificación de entre 67\% y $100 \%$, dependiendo de la especie, aunque es importante considerar que el número de loci evaluados fue menor (solo 6 marcadores). Por el contrario, la transferibilidad de marcadores de $P$. taeda, $P$. merkusii, $P$. resinosa y $P$. densiflora en $P$. kesiya fue considerablemente baja (de $0 \%$ a $20 \%$ ), debido posiblemente a la distancia filogenética de las especies originales con la especie objetivo (Rai et al., 2018). El tamaño de los fragmentos generados en las diferentes especies fue variable (Fig. 1). Todos los marcadores presentaron polimorfismo a través de las especies en las se observó amplificación, con excepción de PtTX4079 y PtTX3063 (Tabla 4). Las diferencias entre los fragmentos obtenidos por un marcador se deben posiblemente al número de unidades de repetición que posee cada uno de ellos, producto de la adición o deleción de dichas unidades.

TABLA 3. Clasificación taxonómica de las especies del género Pinus L. incluidas en el estudio (Gernandt, Geada, Ortiz y Liston, 2005; Kaundun y Lebreton, 2010; Hernández-León, Gernandt, Pérez de la Rosa y Jardón-Barbolla, 2013).

\begin{tabular}{cccc}
\hline Especie & Subgénero & Sección & Subsección \\
\hline Pinus ayacahuite & Strobus & Quinquefoliae & Strobus \\
Pinus cembroides & Strobus & Parrya & Cembroides \\
Pinus devoniana & Pinus & Trifoliae & Ponderosae \\
Pinus hartwegii & Pinus & Trifoliae & Ponderosae \\
Pinus jeffreyi & Pinus & Trifoliae & Ponderosae \\
Pinus lumholtzii & Pinus & Trifoliae & Australes \\
Pinus luzmariae & Pinus & Trifoliae & Australes \\
Pinus patula & Pinus & Trifoliae & Australes \\
Pinus pseudostrobus & Pinus & Trifoliae & Ponderosae \\
Pinus taeda & Pinus & Trifoliae & Australes \\
\hline
\end{tabular}




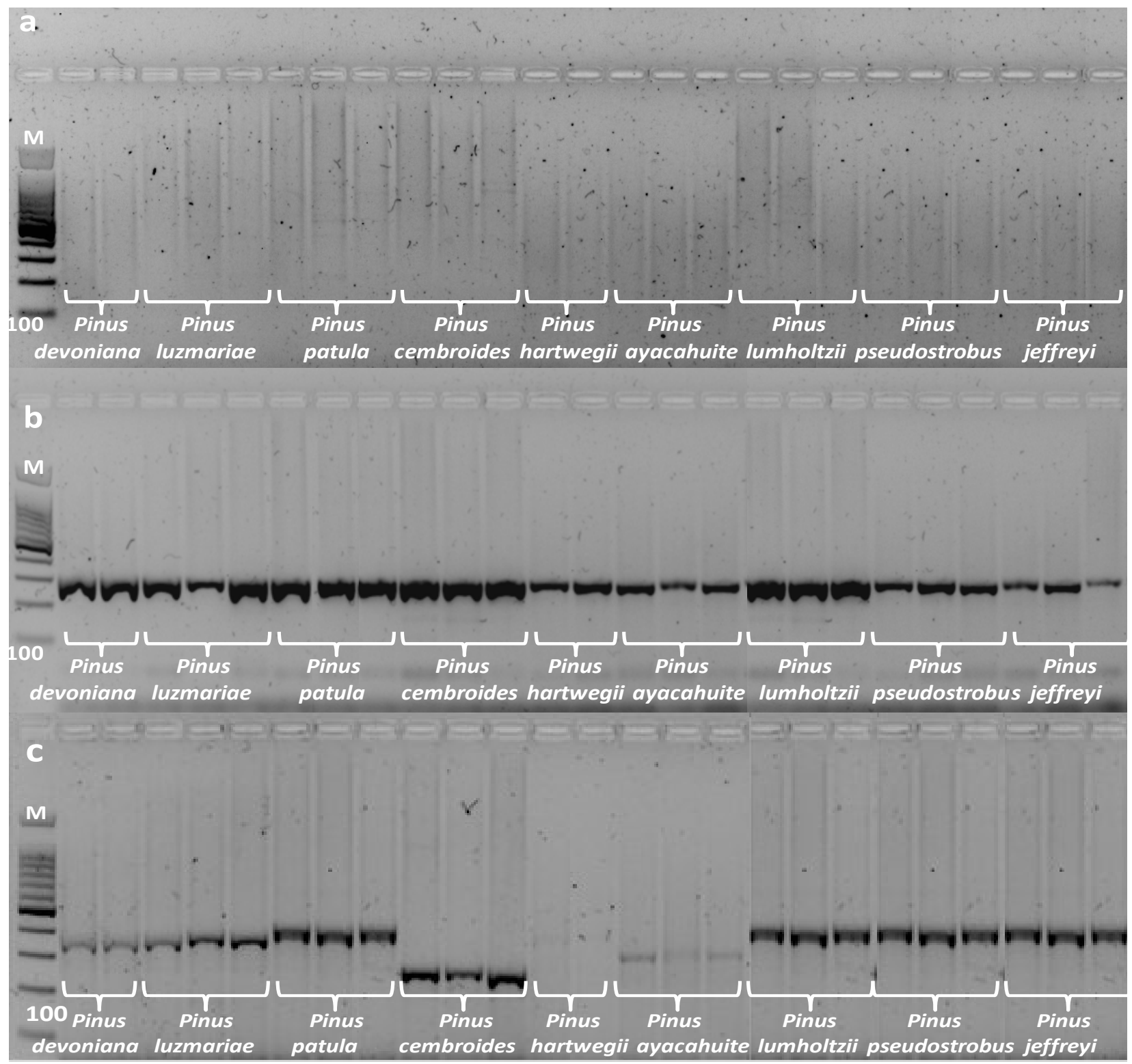

FIGURA 1. Amplificación de microsatélites (SSR) en nueve especies de Pinus L. a) Muestra ausencia de amplificación del marcador PtTX3019 en las nueve especies. b) Muestra presencia de amplificación del marcador PtSIFG_0249 en las nueve especies. c) Muestra presencia y ausencia de amplificación del marcador PtSIFG_4304 y productos con diferente peso molecular.

Los diferentes pesos moleculares también pueden ser resultado de errores de la polimerasa durante el proceso de replicación del ADN o durante la recombinación, obteniéndose cadenas hijas con longitudes desiguales a las parentales (Mason, 2015). Esto significa que alelos que difieren en una sola unidad de repetición están más relacionados por descendencia que alelos que difieren en más de una repetición (Demarchi 2009; Carneiro et al., 2016). Por lo tanto, las especies que se encuentran filogenéticamente más cercanas a la especie en la que se desarrollaron los iniciadores tendrían mayor éxito de transferibilidad que aquellas que se encuentran lejanas (Chapuis y Estoup, 2007). 
TABLA 4. Tamaño observado en pares de bases de 39 marcadores de microsatélites amplificados en nueve especies de Pinus L.

\begin{tabular}{|c|c|c|c|c|c|c|c|c|c|c|}
\hline Marcador & $\begin{array}{l}0 \\
0 \\
8 \\
0 \\
0 \\
\frac{0}{2} \\
\vdots \\
\vdots \\
0\end{array}$ & $\begin{array}{l}\frac{5}{5} \\
\frac{0}{0} \\
\frac{0}{0} \\
\frac{5}{2} \\
\frac{5}{2}\end{array}$ & 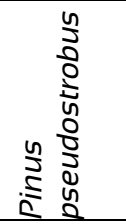 & 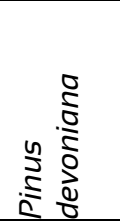 & 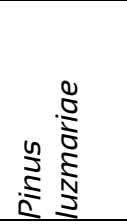 & $\begin{array}{l}\frac{0}{3} \\
\frac{1}{0} \\
\frac{0}{0} \\
0 \\
\frac{1}{2} \\
\frac{1}{1}\end{array}$ & 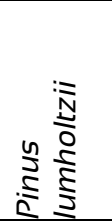 & 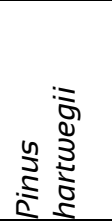 & 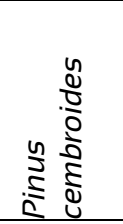 & 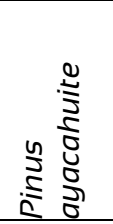 \\
\hline PtRIP_9315 & 207 & 242 & 220 & 200 & 200 & 200 & 200 & 200 & 200 & 200 \\
\hline PtSIFG_0265 & 395 & 370 & 378 & 300 & 300 & 300 & 350 & 310 & 300 & 310 \\
\hline РеTX3029 & 259 & 192 & 200 & 200 & 200 & 200 & 190 & 210 & 210 & 220 \\
\hline РtTX3045 & 255 & 250 & 250 & 200 & 200 & 200 & 250 & 200 & 180 & 100 \\
\hline PtSIFG_0440 & 309 & 300 & 300 & 260 & 260 & 260 & 300 & 260 & 260 & 260 \\
\hline PtSIFG_1018 & 311 & 310 & 320 & 290 & 290 & 290 & 300 & 250 & 250 & 250 \\
\hline PrSIFG_0249 & 297 & 300 & 300 & 290 & 290 & 290 & 300 & 290 & 290 & 290 \\
\hline PtTX4056 & 412 & 400 & 400 & 400 & 400 & 400 & 390 & 400 & 400 & $N / A^{1}$ \\
\hline PtSIFG_0424 & 368 & 370 & 370 & 350 & 350 & 350 & 380 & 370 & 370 & $N / A$ \\
\hline PtRIP_0376 & 180 & 170 & 180 & 160 & 170 & 160 & 180 & 170 & $N / A$ & 170 \\
\hline PtTX2123 & 194 & 190 & 200 & 200 & 200 & 200 & 200 & 200 & $N / A$ & 200 \\
\hline PtSIFG_4304 & 393 & 382 & 374 & 340 & 360 & 400 & 390 & N/A & 260 & 300 \\
\hline PtSIFG_1032 & 282 & 278 & 290 & 300 & 300 & 300 & 390 & 270 & 270 & N/A \\
\hline PtSIFG_1185 & 377 & 390 & 390 & 200 & 200 & 200 & 380 & 200 & 200 & N/A \\
\hline PtRIP_0984 & 216 & 190 & 190 & 150 & 160 & 150 & 190 & 150 & $N / A$ & N/A \\
\hline PtSIFG_0245 & 183 & 180 & 180 & 120 & 120 & 120 & 180 & 120 & $N / A$ & N/A \\
\hline PtRIP_1036 & 184 & 180 & 168 & 100 & 100 & 100 & 170 & 100 & $N / A$ & N/A \\
\hline PtSIFG_4454 & 297 & 318 & 306 & 280 & 280 & 280 & N/A & 280 & $N / A$ & 280 \\
\hline SsrPt_ctg946 & 274 & 270 & 260 & 250 & 250 & 240 & N/A & N/A & 180 & 180 \\
\hline PtRIP_0540 & 204 & 180 & 180 & 180 & 180 & 180 & 170 & N/A & $N / A$ & N/A \\
\hline PtRIP_0134 & 139 & 160 & 160 & 120 & 120 & 100 & N/A & N/A & 170 & N/A \\
\hline PrSIFG_4446 & 115 & 108 & 100 & 100 & 100 & 100 & $N / A$ & 100 & $N / A$ & $N / A$ \\
\hline PtRIP_1035 & 170 & 160 & 160 & 180 & 180 & $N / A$ & 140 & 160 & $N / A$ & N/A \\
\hline РtTX3063 & 259 & 200 & 200 & 200 & 200 & 200 & $N / A$ & 200 & $N / A$ & $N / A$ \\
\hline NZPR1O78 & 336 & $N / A$ & $N / A$ & 280 & 290 & 290 & 320 & 310 & $N / A$ & $N / A$ \\
\hline PtTX4214 & 170 & 268 & 240 & $N / A$ & $N / A$ & 280 & 600 & N/A & 400 & N/A \\
\hline РtTX3047 & 347 & 410 & $N / A$ & 320 & 350 & 320 & 300 & N/A & $N / A$ & N/A \\
\hline PtRIP_0609 & 143 & 180 & $N / A$ & 180 & 180 & 180 & 350 & N/A & $N / A$ & N/A \\
\hline PtRIP_0990 & 207 & $N / A$ & $N / A$ & 120 & 120 & 120 & 100 & $N / A$ & $N / A$ & $N / A$ \\
\hline PtSIFG_02O3 & 110 & 108 & 100 & $N / A$ & $N / A$ & $N / A$ & 100 & N/A & 100 & N/A \\
\hline PtTX4079 & 224 & $N / A$ & $N / A$ & 200 & 200 & $N / A$ & 200 & 200 & $N / A$ & N/A \\
\hline PtTX4228 & 156 & $N / A$ & $N / A$ & 120 & $N / A$ & 120 & 150 & N/A & $N / A$ & $N / A$ \\
\hline PtTX3117 & 196 & 190 & 200 & $N / A$ & $N / A$ & $N / A$ & 200 & N/A & $N / A$ & N/A \\
\hline SsrPt_ctg7141 & 475 & 490 & 480 & $N / A$ & $N / A$ & $N / A$ & N/A & N/A & $N / A$ & N/A \\
\hline PtRIP_0630 & 151 & 160 & 140 & $N / A$ & $N / A$ & $N / A$ & N/A & N/A & $N / A$ & N/A \\
\hline PtSIFG_1062 & 205 & 190 & 190 & $N / A$ & $N / A$ & $N / A$ & N/A & N/A & $N / A$ & N/A \\
\hline PtRIP_1072 & 213 & $N / A$ & $N / A$ & $N / A$ & $N / A$ & $N / A$ & 300 & N/A & $N / A$ & N/A \\
\hline РtTX3027 & 280 & $N / A$ & $N / A$ & $N / A$ & $N / A$ & $N / A$ & N/A & N/A & $N / A$ & N/A \\
\hline PtTX3019 & 215 & $N / A$ & $N / A$ & $N / A$ & $N / A$ & $N / A$ & N/A & N/A & $\mathrm{N} / \mathrm{A}$ & N/A \\
\hline
\end{tabular}

'N/A: no amplificación 
Sin embargo, la transferibilidad entre especies cercanas no siempre ocurre. El concepto filogenético no necesariamente define el éxito de la amplificación interespecífica. En el presente trabajo, se podría esperar que P. patula, P. luzmariae y P. lumboltzii presentaran un mayor porcentaje de transferibilidad ya que pertenecen a la misma subsección (Australes) que P. taeda (Tabla 3). En contraste, $P$. jeffrei, $P$. pseudostrobus y $P$. devoniana, las cuales se encuentran en una subsección diferente a $P$. taeda (Ponderosae), registraron los mayores porcentajes de transferibilidad. Los bajos niveles de transferibilidad entre especies cercanas pueden ser explicados por el fenómeno de recombinación o de mutación en el sitio de unión de los iniciadores. La recombinación actúa mediante arreglos independientes de genes en diferentes cromosomas o por entrecruzamientos entre genes homólogos, contribuyendo a la generación de combinaciones de alelos en nuevos genomas (Salas, 2007). Las mutaciones en los sitios de unión de los cebadores podrían hacer que estos no se unan a la región complementaria y así evitar la amplificación de un fragmento, afectando la estimación de la diversidad genética y la diferenciación de las poblaciones (Chapuis y Estoup, 2007). Por otro lado, la identificación de los fragmentos (alelos) se basa, principalmente en la determinación de su tamaño, sin embargo, no todos los alelos que tienen la misma longitud presentan la misma secuencia. Estos alelos suelen dificultar la identificación de la diversidad alélica ya que pueden tener un origen genealógico diferente o, por el contrario, sobreestimar el flujo genético cuando la tasa de mutación es alta, particularmente cuando se usan en grupos altamente divergentes (Selkoe y Toonen, 2006). Es decir, el éxito de la transferibilidad no depende enteramente de la relación filogenética entre especies, ya que existen procesos que pueden modificar regiones puntuales en el genoma y sobreestimar el polimorfismo o reducir el porcentaje de amplificación inter-específica.

\section{CONCLUSIONES}

Se identificaron entre 12 y 31 marcadores SSR óptimos para el análisis de las especies de pino estudiadas; no obstante, se sugiere evaluar otros marcadores para las especies que registraron menor amplificación ( $P$. ayacabuite $y \quad P$. cembroides). La transferibilidad de estos marcadores representa una alternativa más económica y rápida comparada con el desarrollo de marcadores especieespecíficos, permitiendo la selección de un grupo de marcadores microsatélites para la realización de estudios de diversidad genética en especies del género Pinus y la comparación de los resultados interlaboratorios a través de métodos estandarizados.

\section{RECONOCIMIENTOS}

Este trabajo fue financiado por la Comisión Nacional Forestal (Conafor) a través del proyecto "Fomento y Operación del Subsistema de Recursos Genéticos Forestales dentro del Centro Nacional de Recursos Genéticos (CNRG)"

\section{REFERENCIAS}

Carneiro, M. L., Santini, L., Lima, A., \& de Freitas, C. (2016). Microsatellite markers: what they mean and why are so useful. Genetics and Molecular Biology, 39, 312-328. doi:10.1590/1678-4685GMB-2016-0027

Celiński, K., Pawlaczyk, E. M., Wojnicka-Półtorak, A., Chudzińska, E., \& Prus-Głowacki, W. (2013). Cross-species amplification and characterization of microsatellite loci in Pinus mugo Turra. Biologia, 68, 621-626. doi: 10.2478/s11756-013-0189-z

Chagne, D., Chaumeil, P., Ramboer, A., Collada, C., Guevara, M. A., Cervera, M. T., Vendramin, G. G., Garcia, V., Frigerio, J. M., Echt, C., Richardson, T., \& Plomion, C. (2004). Cross species transferability and mapping of genomic and cDNA SSRs in pines. TAG. Theoretical and Applied Genetics, 109(6), 1204-1214. doi: 10.1007/s00122-004-1683-z

Chapuis, M., P., \& Estoup, A. (2007). Microsatellite null alleles and estimation of population differentiation. Molecular Biology and Evolution, 24(3), 621-31. doi: 10.1093/molbev/msl191

Del Castillo, R. F., Pérez-de la Rosa, J. A., Vargas, G., \& Rivera, R. (2004). Coníferas: En A. J. García-Mendoza, M. J. Ordóñez y M. Briones-Salas (Eds.), Biodiversidad de Oaxaca (pp. 141-158). México: Instituto de Biología, UNAM. Fondo Oaxaqueño para la Conservación de la Naturaleza-World Wildlife Fund. 
Demarchi, D. A. (2009). Microsatélites, distancias genéticas y estructura de poblaciones nativas sudamericanas. Revista Argentina de Antropología Biológica, 1(1), 73-88.

Echt, C. S., Vendramin, G. G., Nelson, C. D., \& Marquardt, P. (1999). Microsatellite DNA as shared genetic markers among conifer species. Canadian Journal of Forest Research, 29, 365-371. doi: 10.1139/cjfr-29-3-365

Echt, C. S., Saha, S., Krutovsky, K. V., Wimalanathan, K., Erpelding, J. E., Liang, C., \& Nelson, C. D. (2011). An annotated genetic map of loblolly pine based. BMC Genetics, 12(17), 1-16. doi: 10.1186/1471-2156-12-17

Freeman, S. \& Herron, J. C. (2002). Análisis Evolutivo. Madrid, España: Pearson Educación.

Feng, Y. H., Yang, Z. Q., Wang, J., Luo, Q. F., \& Li, H. G. (2014). Development and characterization of SSR markers from Pinus massoniana and their transferability to $P$. elliottii, $P$. caribaea and $P$. yunnanensis. Genetics and Molecular Research, 13(1), 1508-1513. doi: $10.4238 / 2014$

Geburek, T., \& Konrad, H. (2008). Why the conservation of Forest Genetic Resources has not worked. Conservation Biology, 22, $267-$ 274. doi: 10.1111/j.1523-1739.2008.00900.x

Gernandt, D. S., Geada, G., Ortiz, S., \& Liston, A. (2005). Phylogeny and Classification of Pinus. Taxon, 54(1), 29-42. doi: $10.2307 / 25065300$

Gernandt, D. S., \& Pérez-de la Rosa, J. A. (2014). Biodiversidad de Pinophyta (coníferas) en México. Revista Mexicana de Biodiversidad, 85, 126-133. doi: 10.7550/rmb.32195

Gutiérrez, E., \& Trejo, I. (2014). Efecto del cambio climático en la distribución potencial de cinco especies. Revista Mexicana de Biodiversidad, 85(1), 179-188.

Hernández-León, S., Gernandt, D. S., Pérez de la Rosa, J. A., \& JardónBarbolla, L. (2013). Phylogenetic Relationships and Species Delimitation in Pinus Section Trifoliae Inferrred from Plastid DNA. PLoS ONE, 8(7), e70501. doi: 10.1371/journal.pone.0070501

Huang, Q. X., Guang, X. C., Kong, H., Guo, Y. L., \& Guo, A. P. (2013) An efficient DNA isolation method for tropical plants. African Journal of Biotechnology, 12(19), 2727-2732. doi: 10.5897/AJB12.524

Kaundun, S. S., \& Lebreton, P. (2010). Taxonomy and systematics of the genus Pinus based on morphological, biogeographical and biochemical characters. Plant Systematics and Evolution, 284, 1-15. doi: 10.1007/s00606-009-0228-y
Lesser, M., Parchman, T., \& Buerkle, C. (2011). Cross-species transferability of SSR loci developed from transciptome sequencing in lodgepole pine. Molecular Ecology Resources, 12(3), 448-455. doi: 10.1111/j.1755-0998.2011.03102.x

Mason, A. S. (2015). SSR Genotyping. En J. Batley (Ed.), Plant Genotyping: Methods and Protocols. Methods in Molecular Biology vol. 1245 (pp. 7789). New York, NY: Humana Press.

Moreno, L. A. (2007) Tamaño efectivo de la población: En L. E. Eguiarte, V. Souza, \& X. Aguirre (comps.), Ecología molecular (pp. 63-86). D.F., México: Comisión Nacional para el Conocimiento y Uso de la Biodiversidad.

Rentaría, A. M. (2007). Breve revisión de los marcadores moleculares: En L. E. Eguiarte, V. Souza, \& X. Aguirre (Comps.), Ecología molecular (pp. 541-566). D.F., México: Comisión Nacional para el Conocimiento y Uso de la Biodiversidad.

Rai, K. C., Ginwal, H. S., \& Saha, R. (2018). Genetic diversity and population structure of Pinus kesiya through trans-specific amplification of nuclear SSR markers. International Journal of Agriculture, Environment and Biotechnology, 11(5), 733-739. doi: 10.30954/0974-1712.10.2018.4

Rocha, M., \& Gasca, J. (2007). Ecología molecular de la conservación: En L. E. Eguiarte, V. Souza, \& X. Aguirre (comps.), Ecología molecular (pp. 251-280). México: Comisión Nacional para el Conocimiento y Uso de la Biodiversidad.

Salas, R. (2007). La recombinación: relevancia evolutiva y métodos de estimación, con énfasis en microorganismos: En L. E. Eguiarte, V. Souza y X. Aguirre (comps.), Ecología molecular (pp. 281-311). D.F., México: Comisión Nacional para el Conocimiento y Uso de la Biodiversidad.

Saghai-Maroof, M. A., Soliman, K. M., Jorgensen, R. A., \& Allard, R. W. (1984). Ribosomal DNA spacer-length polymorphism in barley: Mendelian inheritance, chromosomal location, and population dynamics. Proceedings of the National Academy of Sciences US A, 8(24), 8014-8018. doi: 10.1073/pnas.81.24.8014

Selkoe, K., \& Toonen, R. J. (2006). Microsatellites for ecologists: a practical guide to using and evaluating microsatellite markers. Ecology Letters, 9(5), 615-629. doi: 10.1111/j.14610248.2006.00889.x

Valdés-Infante Herrero, J., Rodríguez, N. N., Bautista, M., Ortiz, M. M., Quiroz, A., Sánchez, L. F., Risterucci, A. M., \& Rohde, W. (2010). Microsatélites desarrollados en guayabo (Psidium guajava L.) y su utilidad para evaluar diversidad en la familia Myrtaceae. Revista Colombiana de Biotecnología, 12, 64-76. 
Villalobos-Arámbula, A. R., Pérez de la Rosa, J. A., Arias, A., \& Rajora, O. P. (2014). Cross-species transferability of eastern white pine (Pinus strobus) nuclear microsatellite markers to five Mexican white pines. Genetics and Molecular Research, 13(3), 7571-7576. doi: $10.4238 / 2014$

Manuscrito recibido el 20 de enero de 2021

Aceptado el 03 de junio de 2021

Publicado el 31 de diciembre de 2021
Este documento se debe citar como:

Sandoval-Padilla, I., Contreras-Toledo, A. R., Guzmán, L. F., Amaro González, B. A., \& Cortés-Cruz, M. (2021). Transferibilidad de marcadores de microsatélites en nueve especies de pinos. Madera y Bosques, 27(3), e2732103. doi: 10.21829/myb.2021.2732103

Madera y Bosques por Instituto de Ecología, A.C. se distribuye bajo una Licencia Creative Commons Atribución-NoComercialCompartirlgual 4.0 Internacional. 